\title{
BMJ Open Diagnosis of urinary tract infection based on symptoms: how are likelihood ratios affected by age? a diagnostic accuracy study
}

\author{
Anne Holm (D) , Volkert Siersma, Gloria Cristina Cordoba (1)
}

To cite: Holm A, Siersma V, Cordoba GC. Diagnosis of urinary tract infection based on symptoms: how are likelihood ratios affected by age? a diagnostic accuracy study. BMJ Open 2021;11:e039871. doi:10.1136/ bmjopen-2020-039871

- Prepublication history for this paper is available online. To view these files, please visit the journal online (http://dx.doi. org/10.1136/bmjopen-2020039871).

Received 28 April 2020 Revised 29 0ctober 2020 Accepted 16 December 2020

Check for updates

(c) Author(s) (or their employer(s)) 2021. Re-use permitted under CC BY-NC. No commercial re-use. See rights and permissions. Published by BMJ.

The Research Unit for General Practice and Section of General Practice, Department of Public Health, University of Copenhagen, Kobenhavn, Denmark

Correspondence to

Dr Anne Holm;

anneholm@sund.ku.dk

\section{ABSTRACT}

Objectives The aim of this study was to investigate the impact of age on the diagnostic properties of typical symptoms of urinary tract infection (UTI) in women presenting in general practice with symptoms suggestive of UTI with significant bacteriuria as the reference standard.

Design Diagnostic accuracy study.

Setting General practice, Copenhagen, Denmark.

Participants Adult 15 years or older. Women presenting in general practice with symptoms suggestive of UTI where urine was collected for investigation.

Primary and secondary outcome measures Accuracy of four symptoms of UTI (dysuria, frequency, urge and abdominal pain) in six different age groups.

Results 90 practices included 1178 adult women. The prevalence of bacteriuria varied between $30 \%$ in women aged $30-44$ years and $67 \%$ in women aged $75-89$ years. The likelihood ratios for dysuria varied between age groups with the best performance in women aged 15-29 (positive likelihood ratio (pLR): 1.62 (1.30-1.94), negative likelihood ratio (nLR): $0.36(0.19-0.54))$ and women aged 30-44 (pLR: 1.74 (1.30-2.17), nLR: 0.48 (0.27-0.68)). Cls included or approximated one for the remaining symptoms in most age groups. When symptoms were combined to calculate post-test probabilities, the combined effect of the varying prevalence of bacteriuria and the varying diagnostic values resulted in a large variation of the posttest probabilities between age groups.

Conclusions The diagnostic value of symptoms of UTI as well as the prevalence of bacteriuria in women presenting to general practice with suspected UTI vary between age groups with considerable clinical implications. Diagnostic studies should take demographics such as age into consideration.

Trial registration number ClinicalTrials.gov: NCT02698332.

\section{BACKGROUND}

Urinary tract infection (UTI) is a common condition in general practice affecting mostly women. ${ }^{12}$ The diagnosis is often established based on symptoms due to lack of fast and precise point-of-care tests in general practice. $^{34}$
Strengths and limitations of this study

A large cohort of 1178 women presenting consecutively in general practice with symptoms suggestive of urinary tract infection.

- All patients were subjected to both index test (clinical history about symptoms) and reference test.

- Blinding between index test and reference was sufficient and the reference test was appropriate.

- A limited number of variables on each patient were collected.

Clinical review bias may be present.

The accuracy of UTI symptoms in determining bacteriuria has been investigated thoroughly. ${ }^{5-7}$ However, the available research does not take into consideration how age affects the diagnostic properties of signs and symptoms. Either the studies include only young women or do not report different age groups separately.

Age is known to affect the diagnostic properties of urine tests. ${ }^{8}$ This could be due to variation in test performance of either the index or the reference test or both across age groups. ${ }^{910}$ The mechanisms are not fully understood and probably vary depending on the test. ${ }^{11}$ The same could be expected to apply to the accuracy of urine symptoms.

The aim of this study was to investigate the impact of age on the diagnostic properties of typical symptoms of UTI in women presenting in general practice with symptoms suggestive of UTI with significant bacteriuria as the reference standard.

\section{METHODS}

\section{Study design and setting}

Prospective diagnostic study in general practice embedded in a cluster randomised controlled trial. The practices in the original study (unpublished) were randomised to 


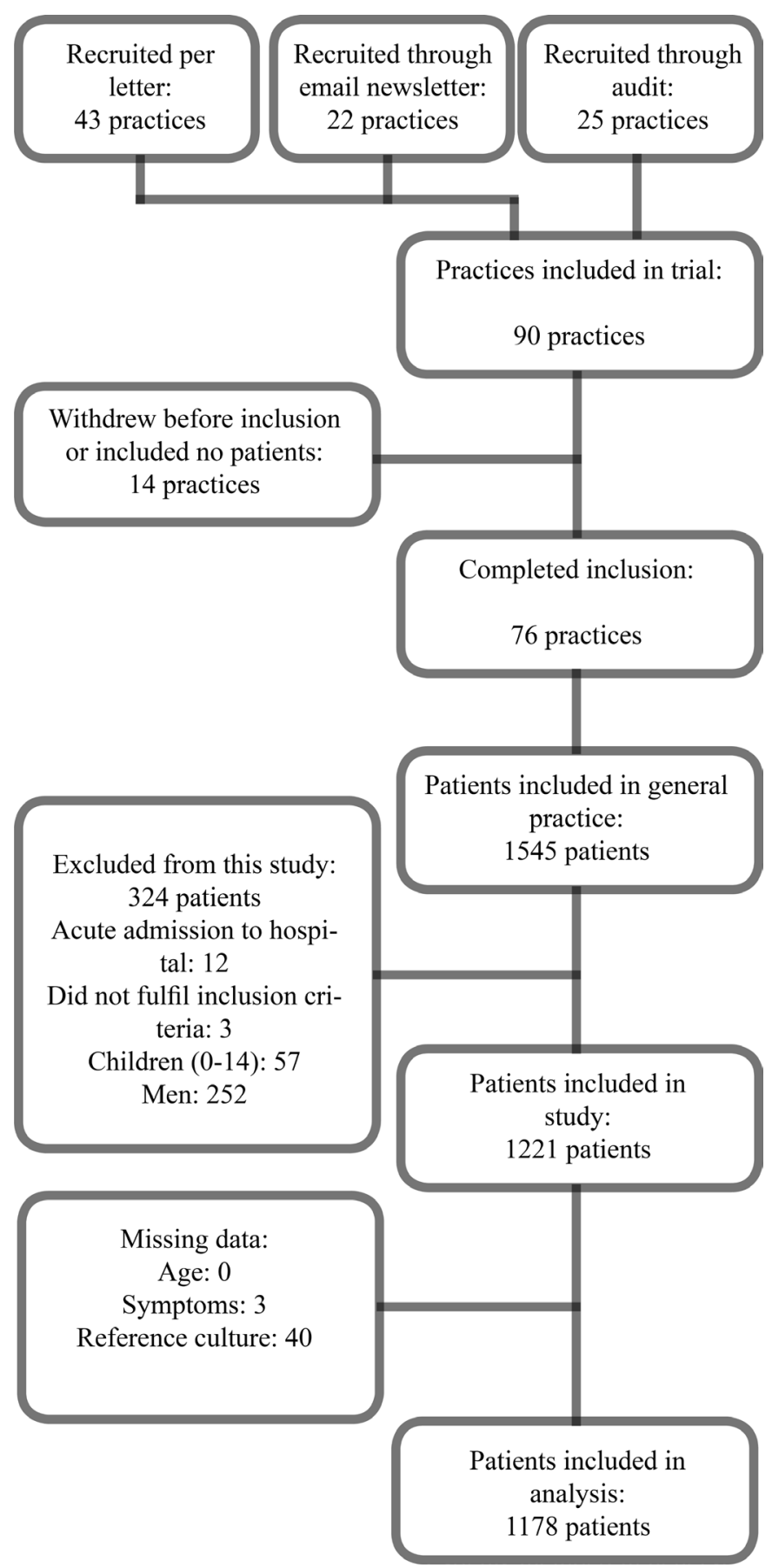

Figure 1 Inclusion and exclusion of practices and patients

either receiving a guideline on how to use point-of-care diagnostic tests or continue usual practice. The intervention did not interfere with registration of symptoms, collection of urine or sending the reference standard.

\section{Recruitment of general practices}

General practices in the Capital Region of Denmark were recruited through three channels; (1) online advertisement in email newsletters to general practice, (2) invitation by post to 200 practices and (3) invitation of 44 general practices already participating in a medical audit project about UTI (figure 1). ${ }^{12}$ Practices were offered a small remuneration and feedback on the quality of diagnosis and treatment of UTI in exchange for participation.

\section{Recruitment of patients}

Data collection took place in March to May 2016. Practices registered symptoms, diagnostics and treatment on the first 20-40 consecutive patients who presented with symptoms suggestive of UTI and where urine was collected for investigation. Patients who had previously been registered in the present project were not registered. Only adult (15 years or older) women who were not admitted acutely to the hospital after evaluation in general practice were included in the analysis for this study.

\section{Data collection}

The practices registered clinical data using a case report form. It contained information on age, sex and if the patients had dysuria, frequency, urge, abdominal pain or 'any other symptom suggestive of UTI' (in this order) as well as the result of the urine culture (online supplemental appendix 1). It was designed following the Audit Project Odense methodology. ${ }^{13}{ }^{14}$ All patients provided a urine sample, which was sent to Hvidovre or Herlev Hospital's microbiological departments. The practice registered the result of the urine culture on the case report form. The options were 'positive: significant growth', 'negative: no significant growth', 'inconclusive' (ie, mixed culture) or 'not performed'.

\section{Culture at the microbiological laboratory (reference standard)}

Urine was sent in a standardised boric acid container to the microbiological departments. Urine samples were analysed on Inoqul A Bi-plate (CHROMagar and blood agar) with $10 \mu \mathrm{L}$ on each half of the agar. Significant growth was defined as growth of $\geq 10^{3} \mathrm{cfu} / \mathrm{mL}$ for Escherichia coli and Staphylococcus saprophyticus, $\geq 10^{4} \mathrm{cfu} / \mathrm{mL}$ for other typical uropathogens and $\geq 10^{5} \mathrm{cfu} / \mathrm{mL}$ for possible uropathogens in accordance with European consensus. ${ }^{15}$ Plates with significant growth of more than two uropathogens were labelled as mixed cultures (inconclusive). Inconclusive cultures were defined as negatives in our analysis since they are usually handled clinically as negatives. Significant bacteriuria has been shown to differentiate patients who recover without treatment from those in need of antibiotic treatment. ${ }^{16}$ However, the clinically relevant cut-off for significant bacteriuria is debated and differs between countries. We chose the cut-off used in Danish microbiological laboratories.

\section{Blinding}

Practices were not aware of the result of the reference culture when symptoms were registered. Likewise, the microbiological departments were not informed about symptoms when analysing the reference culture.

\section{Patient safety}

Patients gave oral informed consent to all diagnostics and treatment in accordance with the Danish Health 
Legislation Act. Patient's data were anonymised before being sent from the practice to the investigators.

\section{Statistical analysis}

Since the sample was based on a cluster randomised controlled trial, it was fixed for this study. Sensitivity (SEN), specificity (SPE), positive likelihood ratio (pLR) and negative likelihood ratio (nLR) were calculated for each age group and reported with exact CIs. The pLR provides an estimate of the increase in odds for having UTI in case a particular symptom is present. The nLR provides an estimate of the decrease in odds of having UTI in case a symptom is absent.

Asking about symptoms in any sequential order can be seen as adding diagnostic tests to each other. The diagnostic value of each symptom can then be added to the previous symptom in an additive process where the posttest probability from the previous symptom serves as the pretest probability of the next symptom. We investigated the utility of combining several symptoms in the order determined by the case report form. The result was illustrated with a Dumbell plot.

As an example; symptom one has a SEN of $80 \%$ and a SPE of $50 \%$, symptom two has a SEN of $60 \%$ and a SPE of $70 \%$. Prevalence of bacteriuria is $50 \%$ (which implies a pretest odds $=1) \cdot \mathrm{pLR} 1=\frac{S E N 1}{1-S P E 1}=\frac{0.80}{1-0.50}=1.60$

$\mathrm{pLR} 2=\frac{S E N 2}{1-S P E 2}=\frac{0.60}{1-0.70}=2$

The post-test probability after presence of symptom one is:

Post-test probability $\quad 1=\frac{\text { Positive posttest odds } 1}{\text { Positive posttest odds } 1+1}=$ $\frac{\text { pretest odds } 1 \cdot p L R 1}{\text { pretest odds } 1 \cdot p L R 1+1}=\frac{1 \cdot 1.60}{1 \cdot 1.60+1}=62 \%($ post-test odds $=1.60)$.

Adding diagnostic values of symptom 2 to symptom one results in:

Post-test probability $2=\frac{\text { Positive posttest odds } 2}{\text { Positive posttest odds } 2+1}=$ $\frac{\text { pretest odds } 2 \cdot p L R 2}{\text { pretest odds } 2 \cdot \text { pLR2+1 }} \frac{1.60 \cdot 2}{1.60 \cdot 2+1}=76 \%$

Statistical analyses were performed using SAS V.9.4 and the Dumbell plot was created in Microsoft Excel 2010.

\section{RESULTS}

Ninety practices in the Capital Region of Denmark consented to participate. Fourteen of these either did not include any patients or withdrew before inclusion.
The 76 remaining practices included 1545 patients of whom 3 were excluded from the original study for not fulfilling inclusion criteria (2 did not have symptoms and 1 did not provide a urine sample). An additional 321 were excluded from the analysis because they did not fulfil the inclusion criteria for this study (see figure 1). Further, 43 patients had missing data leaving 1178 adult women with symptoms suggestive of UTI for analysis.

Table 1 shows the distribution of bacteriuria and symptoms in the six age groups. The 1178 women were evenly distributed in age groups of 15 years each until the age of 89. Only 46 women were 90 years or older. Significant bacteriuria increased from $39 \%$ in the younger women to $67 \%$ in the older women of $75-89$ years. Dysuria was the most common symptom (56\% overall) followed by frequency ( $52 \%$ overall). Urge and abdominal pain were less frequent in all age groups (21\% and 20\%, respectively, overall). The distribution of symptoms was quite similar between age groups.

Table 2 shows the diagnostic values for dysuria, frequency, urge and abdominal pain in the six age groups. Dysuria showed the best diagnostic performance with an overall pLR of 1.39 (1.24-1.54) and nLR of 0.65 (0.56$0.75)$. The likelihood ratios for dysuria varied between age groups with the best performance in women aged 15-29 (pLR: 1.62 (1.30-1.94), nLR: $0.36(0.19-0.54)$ ) and women aged 30-44 (pLR: 1.74 (1.30-2.17), nLR: $0.48(0.27-0.68)$ ). Frequency had an overall pLR of 1.36 (1.20-1.52) and nLR of $0.72(0.62-0.81)$. CIs included or approximated one in all age groups except women aged 30-44 (pLR: 1.85 (1.32-2.37), nLR: 0.53 (0.33-0.72). Urge had an average pLR of $1.44(1.09-1.78)$ and nLR of $0.91(0.85-0.97)$. Variation between age groups was low and CIs involved one in all age groups. Abdominal pain had a negative correlation with bacteriuria with a pLR of $0.63(0.47-0.79)$ and nLR of 1.12 (1.05-1.19). CIs in most age groups involved one for abdominal pain. Figure 2 shows the clinical implications and additive value of symptoms across age groups. In women aged 15-29, absence of dysuria resulted in a probability of bacteriuria of $19 \%$. Presence of dysuria, frequency, urge and absence of abdominal pain resulted in a probability of bacteriuria of $63 \%$ (age 15-29). In women aged 30-44, the pattern

Table 1 The distribution of bacteriuria and symptoms in the six age groups of women presenting to general practice with suspected urinary tract infection.

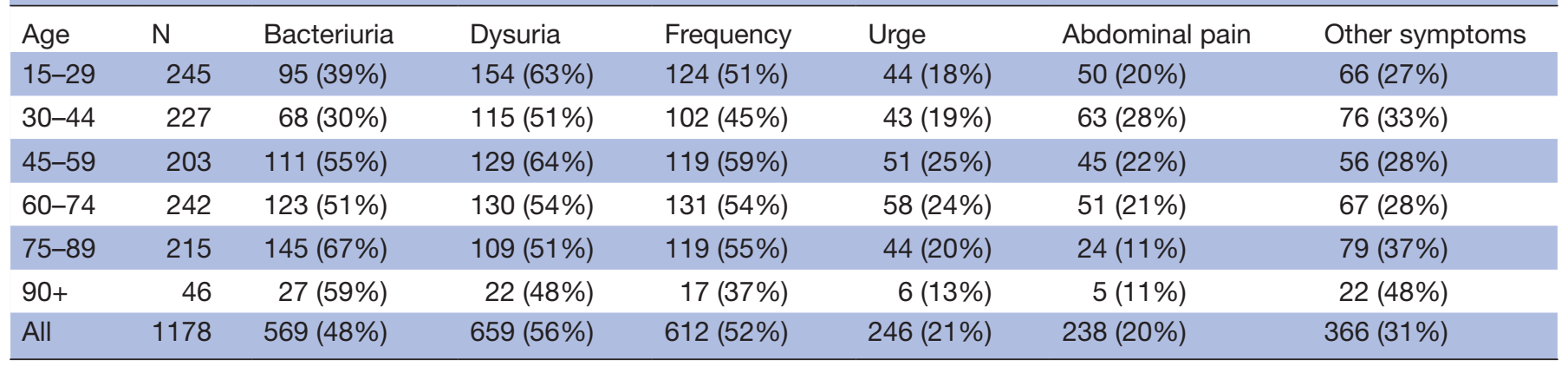


Table 2 Predictive values of dysuria, frequency, urge and abdominal pain for adult (15 years or older) women presenting to general practice with suspected urinary tract infection

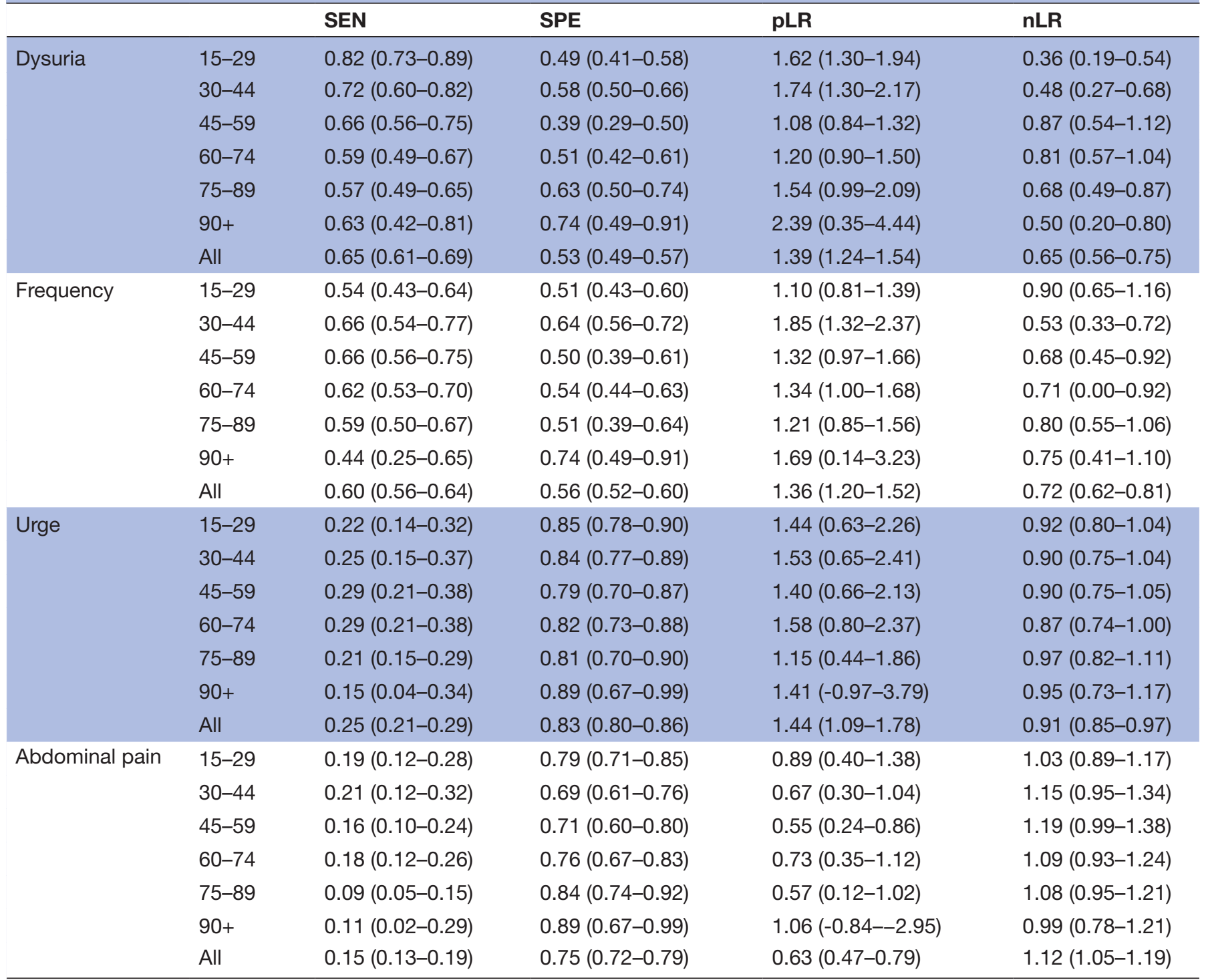

$\mathrm{nLR}$, negative likelihood ratio; pLR, positive likelihood ratio; SEN, sensitivity; SPE, specificity.

resembled that of women aged 15-29. Absence of dysuria resulted in a probability of bacteriuria of $17 \%$. Presence of dysuria, frequency, urge and absence of abdominal pain resulted in a probability of bacteriuria of $71 \%$ (age $30-44)$. Absence of frequency and urge in addition to presence of abdominal pain had limited value in both of the youngest age groups.

In women aged 45-59 and women aged 60-74, dysuria had limited value, but all other symptoms showed some ability to change the probability of bacteriuria. In women aged 45-59 absence of all symptoms (presence of abdominal pain) resulted in a probability of bacteriuria of $27 \%$. Presence of all symptoms (absence of abdominal pain) resulted in a probability of bacteriuria of $74 \%$. In women aged 60-74 absence of all symptoms (presence of abdominal pain) resulted in a probability of bacteriuria of $28 \%$.
Presence of all symptoms (absence of abdominal pain) resulted in a probability of bacteriuria of $74 \%$.

In women aged 75-89, absence of all symptoms (presence of abdominal pain) resulted in a probability of bacteriuria of $39 \%$. Presence of dysuria was able to increase the probability of bacteriuria to $83 \%$. Presence of additional symptoms (absence of abdominal pain) had limited value in this age group. The pattern in women aged 90 years or older resembled that of women aged 75-89.

\section{DISCUSSION}

In this study, the diagnostic properties of dysuria, frequency, urge and abdominal pain in adult women with suspected UTI in general practice varied between age groups. There was a wide variability in the prevalence of bacteriuria. The combined effect of the variability in 


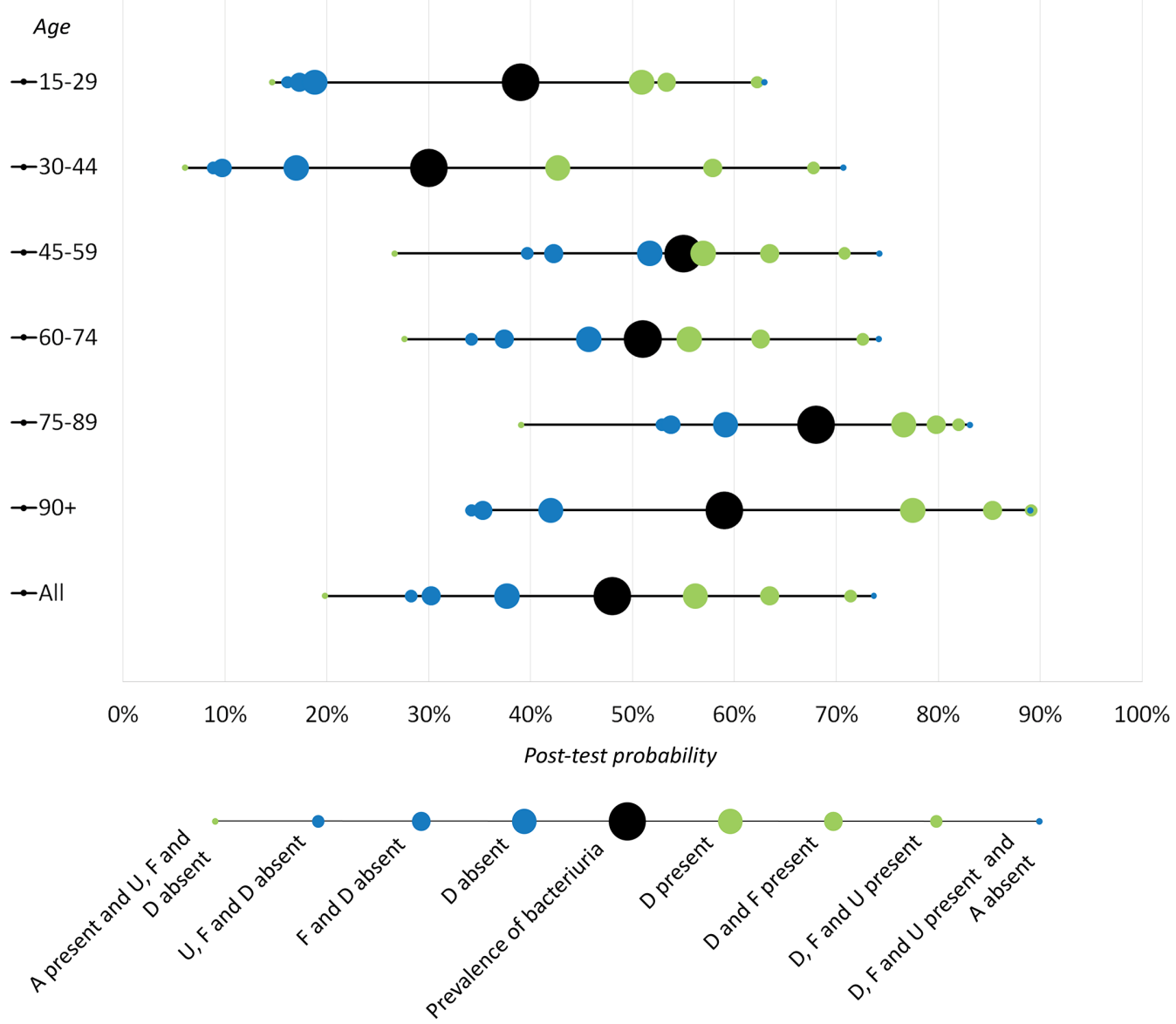

Figure 2 Dumbbell plot of post-test probabilities to illustrate the clinical implications of varying likelyhood ratios (LRs) Symptoms are green when present and blue when absent. Abdominal pain (A) showed a negative association with bacteriuria and is therefore shown on the opposite side. For example, if the patient is 20 years old, her pretest probability of bacteriuria is about $40 \%$. If she has dysuria (D), the probability of bacteriuria increases to around $50 \%$. If she has frequency (F) in addition to $\mathrm{D}$, the probability of bacteriuria slightly increases to a little more than $50 \%$. Note that Cls for all likelihood ratios were wide and the plot can only be used to illustrate how LRs affect post-test probabilities. The exact numbers should be interpreted with caution. A, abdominal pain; D, dysuria; F, frequency; $U$, urge.

the prevalence of bacteriuria and the varying diagnostic values resulted in a large variation in the probability of bacteriuria when symptoms were combined.

The population was representative of the population seeking care in general practice because of urinary tract symptoms. This was possible because the time for data collection was minimal so general practitioners were able to include patients consecutively. However, the simplicity of the data collection method had the drawback that only few symptoms were collected. Thus, we may have overlooked relevant symptoms and were also not able to investigate other relevant demographics than age. ${ }^{9}$

The study was based on data from a cluster randomised trial, but the design was still appropriate for a diagnostic study with minimal bias. The reference standard was centralised, leading to a high quality in the interpretation and minimal review bias. However, clinical review bias was present since the same person collected all clinical information on a patient where 'UTI was suspected'. Thus, individual symptoms may be interdependent leading to errors in diagnostic values, most possibly overestimating SEN and underestimating SPE. ${ }^{9}$ Also, LRs could be inflated in a cohort of patients where UTI is already suspected, since the clinician seeks to confirm their preliminary diagnosis. ${ }^{17}$

The cohort had a sufficient size to provide narrow CIs on the overall estimates of the diagnostic values. However, within the age groups, the uncertainty about the diagnostic values was high, so it is only large heterogeneity that can be identified; smaller heterogeneity cannot be determined with certainty.

A larger study is needed in order to confirm the observed differences. The data were collected in 2016, but it is unlikely, they would be different if they were collected today.

Our definition of UTI (symptoms together with significant bacteriuria) is commonly used, but this definition poses a problem in the older age groups where asymptomatic bacteriuria is more prevalent. ${ }^{18}$ We found an increasing prevalence of bacteriuria with increasing age. Asymptomatic bacteriuria in the elderly is probably only one explanation. Other explanations could be differences in the threshold for seeking care or in the spectrum of differential diagnosis in different age groups. 
The available research on accuracy of UTI symptoms has been conducted with a variety of definitions of significant bacteriuria. ${ }^{6}$ In the year 2000 , the definition of significant bacteriuria in most of Europe was changed from $10^{5}$ $\mathrm{cfu} / \mathrm{mL}$ for all uropathogens to $10^{3} \mathrm{cfu} / \mathrm{mL}$ for common uropathogens, which is the definition used in our study. ${ }^{15}$ Since more than $80 \%$ of all UTI in general practice is caused by common uropathogens, our results are difficult to compare with previous studies. A study from 2006 on women aged 18-70 using the same reference standard as in our study found a pLR of 1.64 and an nLR of 0.52 for moderate-to-severe dysuria (calculated from table 4 in Little $e t a l .{ }^{19}$ This corresponds well with our findings in women below 45 years of age but not for women older than this. Unfortunately, the study does not report the age distribution of the included patients.

Despite the abundant amount of literature on the diagnostic values of UTI symptoms, this is the first study to investigate the impact of age. ${ }^{5-7}$ Previous studies have investigated the difference in diagnostic values of the urine dipstick in different populations but without looking into age specifically. ${ }^{20}$

Knottnerus et al have investigated how likely UTI has to be for Dutch general practitioners to prescribe or withhold antibiotics. ${ }^{21}$ In this Dutch context, a probability below $30 \%$ was sufficient to withhold antibiotics and a probability above $70 \%$ was sufficient to prescribe. If this finding is applied to our results, prescription would possibly be appropriate in older women with only dysuria, while no combination of symptoms would be sufficient for prescription of antibiotics in younger women. Similarly, antibiotics could possibly be withheld for young women without dysuria, while no combination of symptoms could effectively rule out UTI in older women. However, these estimates should be interpreted with caution due to the wide CIs.

The diagnostic value of symptoms of UTI as well as the prevalence of bacteriuria in women presenting to general practice with suspected UTI vary between age groups with considerable clinical implications. First, the prior probability of UTI rises with age. Second, the LR of dysuria is high in young and older women, but seems to decline in middle age. Other classical symptoms of UTI had too broad CIs in this cohort to confirm variation with age. In women younger than 45 years without dysuria, bacteriuria is unlikely, and the general practitioner could consider applying a wait-and-see strategy. In women above 75 with dysuria, bacteriuria is likely and treatment without further diagnostics could be considered. In other age groups, additional symptoms or diagnostics have to be applied in order to diagnose UTI. Diagnostic studies should take demographics such as age into consideration.

Correction notice This article has been corrected since it first published. The provenance and peer review statement has been included.

Twitter Gloria Cristina Cordoba @Cc16Gc

Contributors All authors took part in the design and planning of the study. $\mathrm{AH}$ conducted the study supported by all other authors. AH drafted the first manuscript and all other authors revised the entire manuscript critically and approved the final version for publication. AH is guarantor for the study.

Funding This study was funded by: (a) 2016, University of Copenhagen (grant number: not applicable) and (b) Læge Sofus Carl Emil Friis og Hustru Olga Doris Friis' legat (grant number: not applicable). The funders had no role in study design, data collection and analysis, decision to publish, or preparation of the manuscript.

Competing interests None declared.

Patient and public involvement statement Patients or the public were not involved in the design, or conduct, or reporting, or dissemination plans of our research.

Patient consent for publication Not required.

Ethics approval The study was presented to the ethical committee of Copenhagen and did not require ethical approval (ref. H-15015686).

Provenance and peer review Not commissioned; externally peer reviewed.

Data availability statement Technical appendix, statistical code and dataset can be made available upon reasonable request.

Supplemental material This content has been supplied by the author(s). It has not been vetted by BMJ Publishing Group Limited (BMJ) and may not have been peer-reviewed. Any opinions or recommendations discussed are solely those of the author(s) and are not endorsed by BMJ. BMJ disclaims all liability and responsibility arising from any reliance placed on the content. Where the content includes any translated material, BMJ does not warrant the accuracy and reliability of the translations (including but not limited to local regulations, clinical guidelines, terminology, drug names and drug dosages), and is not responsible for any error and/or omissions arising from translation and adaptation or otherwise.

Open access This is an open access article distributed in accordance with the Creative Commons Attribution Non Commercial (CC BY-NC 4.0) license, which permits others to distribute, remix, adapt, build upon this work non-commercially, and license their derivative works on different terms, provided the original work is properly cited, appropriate credit is given, any changes made indicated, and the use is non-commercial. See: http://creativecommons.org/licenses/by-nc/4.0/.

ORCID iDs

Anne Holm http://orcid.org/0000-0003-3819-3429

Gloria Cristina Cordoba http://orcid.org/0000-0002-2609-0468

\section{REFERENCES}

1 Foxman B. Urinary tract infection syndromes: occurrence, recurrence, bacteriology, risk factors, and disease burden. Infect Dis Clin North Am 2014;28:1-13.

2 Pace WD, Dickinson LM, Staton EW. Seasonal variation in diagnoses and visits to family physicians. Ann Fam Med 2004;2:411-7.

3 Howick J, Cals JWL, Jones C, et al. Current and future use of point-of-care tests in primary care: an international survey in Australia, Belgium, the Netherlands, the UK and the USA. BMJ Open 2014;4:e005611.

4 Turner D, Little P, Raftery J, et al. Cost effectiveness of management strategies for urinary tract infections: results from randomised controlled trial. BMJ 2010;340:c346.

5 Bent S, Nallamothu BK, Simel DL, et al. Does this woman have an acute uncomplicated urinary tract infection? JAMA 2002;287:2701-10.

6 Giesen LGM, Cousins G, Dimitrov BD, et al. Predicting acute uncomplicated urinary tract infection in women: a systematic review of the diagnostic accuracy of symptoms and signs. BMC Fam Pract 2010;11:1-14.

7 Medina-Bombardó D, Jover-Palmer A. Does clinical examination aid in the diagnosis of urinary tract infections in women? A systematic review and meta-analysis. BMC Fam Pract 2011;12:111.

8 Lachs MS, Nachamkin I, Edelstein PH, et al. Spectrum bias in the evaluation of diagnostic tests: lessons from the rapid dipstick test for urinary tract infection. Ann Intern Med 1992;117:135-40.

9 Whiting P, Rutjes AWS, Reitsma JB, et al. Sources of variation and bias in studies of diagnostic accuracy. Ann Intern Med 2004;140:189-202.

10 Biesheuvel C, Irwig L, Bossuyt P. Observed differences in diagnostic test accuracy between patient subgroups: is it real or due to reference standard misclassification? Clin Chem 2007;53:1725-9.

11 Mulherin SA, Miller WC. Spectrum bias or spectrum effect? subgroup variation in diagnostic test evaluation. Ann Intern Med 2002;137:598-603. 
12 Córdoba G, Holm A, Sørensen TM, et al. Use of diagnostic tests and the appropriateness of the treatment decision in patients with suspected urinary tract infection in primary care in Denmark observational study. BMC Fam Pract 2018;19:65.

13 Bentzen N. Medical audit--the APO-method in general practice. Scand J Prim Health Care Suppl 1993;1:13-18.

14 Munck AP, Hansen DG, Lindman A, et al. A Nordic collaboration on medical audit. The apo method for quality development and continuous medical education (CME) in primary health care. Scand $J$ Prim Health Care 1998;16:2-6.

15 Aspevall O, Hallander H, Gant V, et al. European guidelines for urinalysis: a collaborative document produced by European clinical microbiologists and clinical chemists under ECLM in collaboration with ESCMID. Scand J Clin Lab Invest 2000;60:1-96.

16 Monsen TJ, Holm SE, Ferry BM, et al. Mecillinam resistance and outcome of pivmecillinam treatment in uncomplicated lower urinary tract infection in women. APMIS 2014;122:317-23.
17 Mendel R, Traut-Mattausch E, Jonas E, et al. Confirmation bias: why psychiatrists stick to wrong preliminary diagnoses. Psychol Med 2011;41:2651-9.

18 Nicolle LE, Gupta K, Bradley SF, et al. Clinical practice guideline for the management of asymptomatic bacteriuria: 2019 update by the infectious diseases Society of America. Clin Infect Dis 2019:68:1611-5.

19 Little P, Turner S, Rumsby K, et al. Developing clinical rules to predict urinary tract infection in primary care settings: sensitivity and specificity of near patient tests (dipsticks) and clinical scores. Br J Gen Pract 2006;56:606-12.

20 Devillé WLJM, Yzermans JC, van Duijn NP, et al. The urine dipstick test useful to rule out infections. A meta-analysis of the accuracy. BMC Urol 2004;4:4.

21 Knottnerus BJ, Geerlings SE, Moll van Charante EP, et al. Toward a simple diagnostic index for acute uncomplicated urinary tract infections. Ann Fam Med 2013;11:442-51. 